\title{
Edge Contact Forces in Continuous Media
}

\author{
Marco Degiovanni ${ }^{1}$, Alfredo Marzocchi ${ }^{2}$ and Alessandro Musesti ${ }^{3}$ \\ 1 Dipartimento di Matematica e Fisica, Università Cattolica del Sacro Cuore, Via \\ dei Musei 41, I-25121 Brescia, Italy m.degiovanni@dmf .unicatt.it \\ 2 Dipartimento di Matematica, Università di Brescia, Via Valotti 9, I-25133 \\ Brescia, Italy alfredo.marzocchi@ing.unibs.it \\ 3 Dipartimento di Matematica e Fisica, Università Cattolica del Sacro Cuore, Via \\ dei Musei 41, I-25121 Brescia, Italy a.musesti@dmf .unicatt.it
}

\section{Introduction and preliminaries}

In this note we present some results contained in [2] concerning integral properties of second-order powers. More precisely, we introduce the power expended on a subbody by a virtual velocity field, in the spirit of Germain $[6,7]$, but in an axiomatic way similar to that exploited for first-order powers in [11], regarding the power as a function of the subbody and of the velocity field.

As already shown by Dell'Isola and Seppecher [3] and Di Carlo and Tatone [4], higher order powers can be used to describe edge effects, in a way that seems to be simpler as using edge interactions (see Noll and Virga [12] and Forte and Vianello [5]).

Here we want to investigate the above subject paying attention to the regularity of the stress (or hyper-stress) fields, as well as of the subbodies on which the stresses act. In doing this, we first obtain results for finite perimeter subbodies and fields with divergence measure in order to represent a contact power as a surface integral; secondly, since the power is of order two, a further integration by parts is formally possible, leading to subsets of codimension 2 , i.e. edges. To this end, we introduce a subclass of the sets of finite perimeter, called sets with curvature measure, where such integral representation can be written. The result is that edge effects are seen as surface integrals involving curvature and/or density which is singular with respect to the area.

Finally, we find that, as in previous papers $[1,10,11]$, that powers are uniquely determined by their properties on $n$-intervals.

For the proofs of all results cited below, the reader is referred to [2].

In the sequel, $\mathscr{L}^{n}$ will denote the $n$-dimensional Lebesgue outer measure and $\mathscr{H}^{k}$ the $k$-dimensional Hausdorff outer measure on $\mathbb{R}^{n}$. Given a Borel subset $\Omega \subseteq \mathbb{R}^{n}$, we denote with $\mathfrak{B}(\Omega)$ the collection of all Borel subsets of $\Omega$. 
The topological closure, interior and boundary of $E \subseteq \mathbb{R}^{n}$ will be denoted as usual by cl $E$, int $E$ and bd $E$, respectively. Denoting with $B_{r}(x)$ the open ball with radius $r$ centered at $x$, we introduce the measure-theoretic interior of $E$

$$
E_{*}=\left\{x \in \mathbb{R}^{n}: \lim _{r \rightarrow 0^{+}}\left(r^{-n} \mathscr{L}^{n}\left(B_{r}(x) \backslash E\right)\right)=0\right\}
$$

and the measure-theoretic boundary of $E$

$$
\partial_{*} E=\mathbb{R}^{n} \backslash\left(E_{*} \cup\left(\mathbb{R}^{n} \backslash E\right)_{*}\right),
$$

which are both Borel subsets of $\mathbb{R}^{n}$. We say that $E \subseteq \mathbb{R}^{n}$ is normalized, if $E_{*}=E$.

Let now $\Omega$ be a Borel subset of $\mathbb{R}^{n}$. We denote by $\mathfrak{M}(\Omega)$ the set of Borel measures $\mu: \mathfrak{B}(\Omega) \rightarrow[0,+\infty]$ finite on compact subsets of $\Omega$ and by $\mathcal{L}_{l o c,+}^{p}(\Omega)$, $p \in[1,+\infty]$, the set of Borel functions $h: \Omega \rightarrow[0,+\infty]$ such that

$$
\int_{K} h^{p} d \mathscr{L}^{n}<+\infty \quad(p<+\infty), \quad \underset{K}{\operatorname{essup}} h<+\infty \quad(p=+\infty)
$$

for every compact subset $K \subseteq \Omega$.

Definition 1. A full grid $G$ is an ordered triple

$$
G=\left(x_{0},\left(e_{1}, \ldots, e_{n}\right), \widehat{G}\right),
$$

where $x_{0} \in \mathbb{R}^{n},\left(e_{1}, \ldots, e_{n}\right)$ is a positively oriented orthonormal basis in $\mathbb{R}^{n}$ and $\widehat{G}$ is a Borel subset of $\mathbb{R}$ with $\mathscr{L}^{1}(\mathbb{R} \backslash \widehat{G})=0$.

If $G_{1}, G_{2}$ are two full grids, we write $G_{1} \subseteq G_{2}$ if $\widehat{G}_{1} \subseteq \widehat{G}_{2}$ and they share the point $x_{0}$ and the list $\left(e_{1}, \ldots, e_{n}\right)$.

Definition 2. We denote by $\mathrm{Sym}_{2}$ the finite-dimensional linear space of all symmetric bilinear forms on $\mathbb{R}^{n}$.

Definition 3. We denote with $\mathcal{R}$ the class of open n-intervals $I$ such that $\operatorname{cl} I \subseteq \Omega$.

Definition 4. Let $G=\left(x_{0},\left(e_{1}, \ldots, e_{n}\right), \widehat{G}\right)$ be a full grid. A subset $M$ of $\mathbb{R}^{n}$ is said to be a $G$-interval, if

$$
M=\left\{x \in \mathbb{R}^{n}: a_{j}<\left(x-x_{0}\right) \cdot e_{j}<b_{j} \forall j=1, \ldots, n\right\}
$$

for some $a_{1}, b_{1}, \ldots, a_{n}, b_{n} \in \widehat{G}$. We set

$$
\mathcal{M}_{G}=\left\{M \subseteq \mathbb{R}^{n}: M \text { is a } G \text {-interval with } \operatorname{cl} M \subseteq \Omega\right\} .
$$

Definition 5. Let $\mathcal{A} \subseteq \mathcal{R}$. We say that $\mathcal{A}$ contains almost all of $\mathcal{R}$, if for every $x_{0} \in \mathbb{R}^{n}$ and every positively oriented orthonormal basis $\left(e_{1}, \ldots, e_{n}\right)$ in $\mathbb{R}^{n}$ there exists a full grid

$$
G=\left(x_{0},\left(e_{1}, \ldots, e_{n}\right), \widehat{G}\right)
$$

such that $\mathcal{M}_{G} \subseteq \mathcal{A}$. 


\section{Second-order powers}

We give below our main definition.

Definition 6. Let $\mathcal{A}$ be a subset of $\mathcal{R}$ containing almost all of $\mathcal{R}$. We say that a function $P: \mathcal{A} \times C^{\infty}(\Omega) \rightarrow \mathbb{R}$ is a second-order power, if the following properties hold:

(a) for every $v \in C^{\infty}(\Omega), P(\cdot, v)$ is countably $*$-additive, i.e.

$$
P\left(\left(\bigcup_{i \in \mathbb{N}} M_{i}\right)_{*}, v\right)=\sum_{i \in \mathbb{N}} P\left(M_{i}, v\right)
$$

for every disjoint sequence $\left(M_{i}\right) \in \mathcal{A}$ such that $\left(\bigcup_{i \in \mathbb{N}} M_{i}\right)_{*} \in \mathcal{A}$;

(b) for every $M \in \mathcal{A}, P(M, \cdot)$ is linear;

(c) there exist $\mu_{0}, \mu_{1}, \mu_{2} \in \mathfrak{M}(\Omega)$ such that for every $M \in \mathcal{A}, v \in C^{\infty}(\Omega)$

$$
|P(M, v)| \leqslant \int_{M}|v(x)| d \mu_{0}(x)+\int_{M}|\nabla v(x)| d \mu_{1}(x)+\int_{M}|\nabla \nabla v(x)| d \mu_{2}(x) .
$$

Definition 7. We will call first-order power a second-order power with $\mu_{2}=$ 0 , and power with order 0 a first-order power with $\mu_{1}=0$.

Remark 1. Let $M \in \mathcal{R}$; then it is easy to prove that for every full grid $G$ there exists a disjoint sequence $\left(M_{i}\right) \subseteq \mathcal{M}_{G}$ such that

$$
\left(\bigcup_{i \in \mathbb{N}} M_{i}\right)_{*}=M
$$

Moreover, one could replace (a) by the following weaker assumption:

(a') for every $v \in C^{\infty}(\Omega)$ and for every full grid $G$,

$$
P\left(\left(\bigcup_{i \in \mathbb{N}} M_{i}\right)_{*}, v\right)=\sum_{i \in \mathbb{N}} P\left(M_{i}, v\right)
$$

whenever $\left(M_{i}\right) \in \mathcal{A} \cap \mathcal{M}_{G}$ is a disjoint sequence such that $\left(\bigcup_{i \in \mathbb{N}} M_{i}\right)_{*} \in \mathcal{A}$.

Remark 2. One can also consider powers $P(M, \mathbf{v})$, where the $\mathbf{v}$ takes values in $\mathbb{R}^{N}, N \geqslant 1$ and define the corresponding power by linearity.

Our first goal is to establish a representation formula for a second-order power. This is not a matter of routine, since $P(M, v)$ depends not only on $v$ and hence is not merely a linear functional on the velocity field. 
Theorem 1. Let $P$ be a second-order power.

Then, there exist bounded Borel maps $A_{0}: \Omega \rightarrow \mathbb{R}, A_{1}: \Omega \rightarrow\left(\mathbb{R}^{n}\right)^{*}$, $A_{2}: \Omega \rightarrow \mathrm{Sym}_{2}$ such that for every $M \in \mathcal{A}, v \in C^{\infty}(\Omega)$

$P(M, v)=\int_{M} A_{0}(x) v(x) d \mu_{0}(x)+\int_{M}\left\langle A_{1}(x), \nabla v(x)\right\rangle d \mu_{1}(x)+\int_{M}\left\langle A_{2}(x), \nabla \nabla v(x)\right\rangle d \mu_{2}(x)$.

Moreover, each $A_{j}$ is uniquely determined $\mu_{j}$-a.e.

The following is a form of converse of the previous theorem.

Proposition 1. Let $\mu_{0}, \mu_{1}, \mu_{2} \in \mathfrak{M}(\Omega)$ and $A_{0}, A_{1}, A_{2}$ as above be Borel and bounded.

Then there exists a set $\mathcal{A} \subseteq \mathcal{R}$ containing almost all of $\mathcal{R}$ such that the function $P: \mathcal{A} \times C^{\infty}(\Omega) \rightarrow \mathbb{R}$ defined as

$P(M, v)=\int_{M}\left\langle A_{0}(x), v(x)\right\rangle d \mu_{0}(x)+\int_{M}\left\langle A_{0}(x), \nabla v(x)\right\rangle d \mu_{1}(x)+\int_{M}\left\langle A_{2}(x), \nabla \nabla v(x)\right\rangle d \mu_{2}(x)$ is a second-order power.

Now we turn to a similar representation formula on Borel subsets of $\Omega$.

Definition 8. Let $\eta \in \mathfrak{M}(\Omega)$. We set

$$
\mathcal{B}_{\eta}=\left\{M \subseteq \mathbb{R}^{n}: M=M_{*}, \operatorname{cl} M \subseteq \Omega, \eta\left(\partial_{*} M\right)=0\right\} .
$$

Theorem 2. Let $P$ be a second-order power. Let $A_{j}(j=0,1,2)$ as in Theorem 1 .

Then there exists $\eta \in \mathfrak{M}(\Omega)$ such that the function $\tilde{P}: \mathcal{B}_{\eta} \times C^{\infty}(\Omega) \rightarrow \mathbb{R}$ defined as

$\tilde{P}(M, v)=\int_{M} A_{0}(x) v(x), d \mu_{0}(x)+\int_{M}\left\langle A_{1}(x), \nabla v(x)\right\rangle d \mu_{1}(x)+\int_{M}\left\langle A_{2}(x), \nabla \nabla v(x)\right\rangle d \mu_{2}(x)$

is an extension of $P$ which satisfies (a), (b) and (c) of Definition 6 on $\mathcal{B}_{\eta}$.

\section{Decomposition of powers}

Up to here, the definitions and assumptions made imply that the power $P$ behaves as an integral on the subbodies, but they do not imply, for example, that the power can be represented as a surface integral, as it is often the case in Continuum Mechanics. Our next definition will precise these features.

Definition 9. A second-order power $P$ is said to be weakly balanced, if there exists $\nu \in \mathfrak{M}(\Omega)$ such that

$$
\forall M \in \mathcal{A}, \forall v \in C_{c}^{\infty}(M): \quad|P(M, v)| \leqslant \int_{M}|v| d \nu .
$$


In particular, $P$ is said to be a contact power, if

$$
\forall M \in \mathcal{A}, \forall v \in C_{c}^{\infty}(M): \quad P(M, v)=0,
$$

namely if it is weakly balanced with $\nu=0$.

A power $P$ of order 0 is said to be a body power.

Note that a body power is always weakly balanced, by choosing trivially $\nu=$ $\mu_{0}$.

Theorem 3. Let $P$ be a weakly balanced second-order power and let $A_{j}, j=$ $0,1,2$, be as in Theorem 1 .

Then the following facts hold:

(a) there exists a bounded Borel function $B: \Omega \rightarrow \mathbb{R}$ such that for every $v \in C_{c}^{\infty}(\Omega)$

$$
\int_{M}\left\langle A_{0}(x), v(x)\right\rangle d \mu_{0}(x)+\int_{M}\left\langle A_{1}(x), \nabla v(x)\right\rangle d \mu_{1}(x)+\int_{M}\left\langle A_{2}(x), \nabla \nabla v(x)\right\rangle d \mu_{2}(x)=\int_{\Omega} B(x) v(x) d \nu(x) ;
$$

moreover, $B$ is uniquely determined $\nu$-a.e.;

(b) taking $\mathcal{B}_{\eta}$ as in Theorem 2, we have

$$
\forall M \in \mathcal{B}_{\eta}, \forall v \in C_{c}^{\infty}(M): \quad P(M, v)=\int_{M} B(x) v(x) d \nu(x) .
$$

Let now $P$ be a weakly balanced second-order power, let $\mu_{j}, A_{j}, 0 \leqslant j \leqslant k$, be as in Theorem 1 and let $\nu, B$ be as in Theorem 3. According to Proposition 1 we can define, for a suitable class $\mathcal{A}$ containing almost all of $\mathcal{R}$, two powers $P_{b}, P_{c}: \mathcal{A} \times C^{\infty}(\Omega) \rightarrow \mathbb{R}$ by

$$
\begin{aligned}
& P_{b}(M, v):=\int_{M} B(x) v(x) d \nu(x), \\
& P_{c}(M, v):=P(M, v)-\int_{M} B(x) v(x) d \nu(x) .
\end{aligned}
$$

It is readily seen that $P_{b}$ is a body power and $P_{c}$ a second-order contact power. Of course, we have $P=P_{b}+P_{c}$.

Definition 10. $P_{b}$ is said to be the body part of $P$ and $P_{c}$ the contact part of $P$.

\section{First-order contact powers}

Let $P$ be a first-order contact power such that $(c)$ of Definition 6 holds with $\mu_{1}$ absolutely continuous with respect to the Lebesgue outer measure. We set $\eta=\mu_{0}$. 
According to Theorems 1 and 3 , there exist a bounded Borel function $a: \Omega \rightarrow \mathbb{R}$ and $T \in L_{l o c}^{1}\left(\Omega ; \mathbb{R}^{n}\right)$ such that

$$
\begin{aligned}
\forall M \in \mathcal{B}_{\eta}, \forall v \in C^{\infty}(\Omega): & P(M, v)=\int_{M} a v d \eta+\int_{M} T \cdot \nabla v d \mathscr{L}^{n}, \\
\forall v \in C_{c}^{\infty}(\Omega): & \int_{\Omega} a v d \eta+\int_{\Omega} T \cdot \nabla v d \mathscr{L}^{n}=0 .
\end{aligned}
$$

Moreover, $a$ is uniquely determined $\eta$-a.e. and $T$ is uniquely determined $\mathscr{L}^{n}$ a.e.

We now briefly recall the concept of outer normal to the measure-theoretic boundary of a set. Let $M \subseteq \mathbb{R}^{n}$ and $x \in \partial_{*} M$. We denote by $\mathbf{n}^{M}(x) \in \mathbb{R}^{n}$ a unit vector such that

$$
\begin{gathered}
\mathscr{L}^{n}\left(\left\{\xi \in B_{r}(x) \cap M:(\xi-x) \cdot \mathbf{n}^{M}(x)>0\right\}\right) / r^{n} \rightarrow 0, \\
\mathscr{L}^{n}\left(\left\{\xi \in B_{r}(x) \backslash M:(\xi-x) \cdot \mathbf{n}^{M}(x)<0\right\}\right) / r^{n} \rightarrow 0
\end{gathered}
$$

as $r \rightarrow 0^{+}$. No more than one such vector can exist. Setting $\mathbf{n}^{M}(x)=0$ elsewhere, we can consider the map $\mathbf{n}^{M}: \partial_{*} M \rightarrow \mathbb{R}^{n}$, which is called the unit outer normal to $M$. It turns out that $\mathbf{n}^{M}$ is Borel and bounded.

Whenever $\mathscr{H}^{n-1}\left(\partial_{*} M\right)<+\infty$, we say that $M$ is a set with finite perimeter. In that case it is well known that $\mathbf{n}^{M}(x) \neq 0$ for $\mathscr{H}^{n-1}$-a.e. $x \in \partial_{*} M$ and the Gauss-Green theorem holds.

Now we define a suitable subclass of $\mathcal{B}_{\eta}$ which allows to give a representation formula for a first-order contact power involving only the measuretheoretic boundary of the subbodies. We refer to $[14,1]$ for a discussion about this class.

Definition 11. For $h \in \mathcal{L}_{\text {loc },+}^{1}(\Omega)$ we set

$$
\mathcal{M}_{h \eta}=\left\{M \in \mathcal{B}_{\eta}: \mathscr{H}^{n-1}\left(\partial_{*} M\right)<+\infty, \int_{\partial_{*} M} h d \mathscr{H}^{n-1}<+\infty\right\} .
$$

We are now in position to state the boundary representation formula for firstorder contact power. We refer to it as the Cauchy's Stress Theorem, since it states the linearity of the stress with respect to the normal.

Theorem 4 (Cauchy's Stress Theorem). There exists $h \in \mathcal{L}_{\text {loc },+}^{1}(\Omega)$ such that

$$
\forall M \in \mathcal{M}_{h \eta}, \forall v \in C^{\infty}(\Omega): \quad P(M, v)=\int_{\partial_{*} M} v T \cdot \mathbf{n}^{M} d \mathscr{H}^{n-1} .
$$

\section{Second-order contact powers}

Now we want to study in more detail second-order contact powers and the possibility of representing them as surface integrals. 
Throughout this section, we assume that $P$ is a second-order contact power such that $(c)$ of Definition 6 holds with $\mu_{1}$ and $\mu_{2}$ absolutely continuous with respect to the Lebesgue outer measure. We set $\eta=\mu_{0}$.

According to Theorems 1 and 3 , there exist a bounded Borel function $a: \Omega \rightarrow \mathbb{R}, B \in L_{l o c}^{1}\left(\Omega ; \mathbb{R}^{n}\right)$ and $\mathrm{C} \in L_{l o c}^{1}\left(\Omega ; \operatorname{Sym}_{2}\right)$ such that

$$
\begin{gathered}
\forall M \in \mathcal{B}_{\eta}, \forall v \in C^{\infty}(\Omega): \quad P(M, v)=\int_{M} a v d \eta+\int_{M} B \cdot \nabla v d \mathscr{L}^{n}+\int_{M} \mathrm{C} \cdot \nabla \nabla v d \mathscr{L}^{n}, \\
\forall v \in C_{c}^{\infty}(\Omega): \quad \int_{\Omega} a v d \eta+\int_{\Omega} B \cdot \nabla v d \mathscr{L}^{n}+\int_{\Omega} \mathrm{C} \cdot \nabla \nabla v d \mathscr{L}^{n}=0
\end{gathered}
$$

Moreover, $a$ is uniquely determined $\eta$-a.e. and $B, C$ are uniquely determined $\mathscr{L}^{n}$-a.e. When the distribution $\operatorname{div} \mathrm{C}$ is a function, we have a representation of $P(M, v)$ as a surface integral.

Theorem 5. Assume that $\operatorname{div} C \in L_{l o c}^{1}\left(\Omega, \mathbb{R}^{n}\right)$. Then there exists $h \in \mathcal{L}_{\text {loc },+}^{1}(\Omega)$ such that

$$
P(M, v)=\int_{\partial_{*} M}\left[v(B-\operatorname{div} \mathrm{C}) \cdot \mathbf{n}^{M}+\nabla v \cdot \mathbf{C} \mathbf{n}^{M}\right] d \mathscr{H}^{n-1}
$$

for every $v \in C^{\infty}(\Omega)$ and $M \in \mathcal{M}_{h \eta}$.

A very remarkable feature of our approach is that the condition $\operatorname{div} C \in$ $L_{\text {loc }}^{1}\left(\Omega, \mathbb{R}^{n}\right)$, mentioned in the above theorem, has a counterpart in terms of the power $P$, as we will show in Theorem 6 below.

This is quite interesting, since assumptions made on $P$ are in general more 'physical' than those made on its densities.

To state this, we need a definition and a proposition.

Definition 12. Let $G=\left(x_{0},\left(e_{1}, \ldots, e_{n}\right), \widehat{G}\right)$ be a full grid and $M \in \mathcal{M}_{G}$ of the form

$$
M=\left\{x \in \mathbb{R}^{n}: a_{j}<\left(x-x_{0}\right) \cdot e_{j}<b_{j}, j=1, \ldots, n\right\},
$$

where $a_{1}, b_{1}, \ldots, a_{n}, b_{n} \in \widehat{G}$. Whenever $1 \leqslant j \leqslant n$ and $a_{j} \leqslant \alpha<\beta \leqslant b_{j}$, we set

$$
M_{\alpha, \beta}^{(j)}=\left\{x \in \mathbb{R}^{n}: \alpha<\left(x-x_{0}\right) \cdot e_{j}<\beta, a_{i}<\left(x-x_{0}\right) \cdot e_{i}<b_{i} \quad \forall i \neq j\right\} .
$$

We simply write $M_{\beta}^{(j)}$ in the case $\alpha=a_{j}$.

Proposition 2. Let $\mathcal{M}_{G} \subseteq \mathcal{A}, M \in \mathcal{M}_{G}$ be represented as in (7), $v \in$ $C_{c}^{\infty}(M)$ and $1 \leqslant j \leqslant n$. Then $M_{\beta}^{(j)} \in \mathcal{M}_{G}$ for $\mathscr{L}^{1}$-a.e. $\beta \in\left(a_{j}, b_{j}\right]$ and the map

$$
\left\{\beta \mapsto P\left(M_{\beta}^{(j)}, v\right)\right\}
$$

belongs to $L^{\infty}\left(a_{j}, b_{j}\right)$ for every $v \in C_{c}^{\infty}(\Omega)$. 
At this point we have

Theorem 6. We have that $\operatorname{div} C \in L_{\text {loc }}^{1}\left(\Omega, \mathbb{R}^{n}\right)$ if and only if there exist $h \in$ $\mathcal{L}_{\text {loc, }+}^{1}(\Omega)$ such that

$$
\left|\int_{a_{j}}^{b_{j}} P\left(M_{\beta}^{(j)}, v\right) d \beta\right| \leqslant \int_{M}|v| h d \mathscr{L}^{n}
$$

for every $\mathcal{M}_{G} \subseteq \mathcal{A}, M \in \mathcal{M}_{G}, v \in C_{c}^{\infty}(M)$ and $j=1, \ldots, n$. In this case, we have $|B-2 \operatorname{div} C| \leqslant h$ on $\mathscr{L}^{n}$-a.a. of $\Omega$.

\section{Boundary representation with edges}

Now we come to the most interesting application of second-order powers, namely, the possibility of having a representation formula on edges, or simply sets with non-smooth normal. Roughly speaking, this is made possible by the gradient term in eq. (6), which allows a further integration by parts.

To do this, we need to introduce a new class of sets.

Definition 13. Let $M$ be a normalized set with finite perimeter. We say that $M$ is a set with curvature measure, if there exist $\lambda_{M} \in \mathfrak{M}\left(\partial_{*} M\right)$ with $\lambda\left(\partial_{*} M\right)<+\infty$ and a Borel tensor field $\mathrm{U}: \partial_{*} M \rightarrow \operatorname{Sym}_{2}$ with $|\mathrm{U}(x)|=1$ for $\lambda_{M}$-a.e. $x \in \partial_{*} M$, such that

$$
-\int_{\partial_{*} M}\left[-(\operatorname{div} \mathrm{C}) \cdot \mathbf{n}^{M}+\left((\nabla \mathrm{C}) \mathbf{n}^{M} \mathbf{n}^{M}\right) \cdot \mathbf{n}^{M}\right] d \mathscr{H}^{n-1}=\int_{\partial_{*} M} \mathrm{C} \cdot \mathrm{U} d \lambda_{M}
$$

for every $\mathrm{C} \in C_{c}^{\infty}\left(\mathbb{R}^{n} ; \mathrm{Sym}_{2}\right)$. It turns out that $\lambda_{M}$ is uniquely determined and $\mathrm{U}$ is uniquely determined $\lambda_{M}$-a.e.

For $h \in \mathcal{L}_{\text {loc, }+}^{1}(\Omega)$ we set

$$
\mathcal{C}_{h \eta}=\left\{M \in \mathcal{M}_{h \eta}: M \text { has curvature measure and } \int_{\partial_{*} M} h d \lambda_{M}<+\infty\right\} .
$$

Remark 3. One can prove that the elements of $\mathcal{R}$ are sets with curvature measure. Indeed, since on each face the term $\left[-(\operatorname{div} C) \cdot \mathbf{n}^{M}+\left((\nabla C) \mathbf{n}^{M} \mathbf{n}^{M}\right)\right.$. $\left.\mathbf{n}^{M}\right]$ is a surface divergence, it turns out that $\lambda_{M}$ is the Hausdorff measure $\mathscr{H}^{n-2}$ restricted to the edges, and $\mathbf{U}=\mathbf{n}^{M} \otimes \mathbf{N}+\mathbf{N} \otimes \mathbf{n}^{M}$, where $\mathbf{N}$ is the normal to the edge in the hyperplane of the surface.

We are now ready to perform the last integration by parts. In doing this, however, we notice that the normal derivative of $v$ cannot be dropped, since it corresponds to a field of doublets assigned on the boundary.

We also want to let line integrals appear as surface integrals, with respect to a singular measure.

Since the formal integration by parts puts into account the symmetric gradient of a tensor, let us briefly recall its definition. 
Definition 14. Let $\mathrm{C} \in L_{l o c}^{1}\left(\Omega, \operatorname{Sym}_{2}\right)$. We define the symmetric gradient of $\mathrm{C}$ by setting $\left(\nabla^{s} \mathrm{C}\right)$ uvw on $\Omega$ as

$$
\left\langle\left(\nabla^{s} \mathrm{C}\right) u v w, \varphi\right\rangle=\frac{1}{3} \int_{\Omega}[(\mathrm{C} v \cdot w)(\nabla \varphi \cdot u)+(\mathrm{C} u \cdot w)(\nabla \varphi \cdot v)+(\mathrm{C} u \cdot v)(\nabla \varphi \cdot w)] d \mathscr{L}^{n}
$$

for every $\varphi \in C_{c}^{\infty}(\Omega)$. The function $\left\{(u, v, w) \mapsto\left(\nabla^{s} \mathrm{C}\right) u v w\right\}$ is 3-linear and symmetric; moreover, it holds $((\nabla \mathrm{C}) u u) \cdot u=\left(\nabla^{s} \mathrm{C}\right)$ uuu for every $u \in \mathbb{R}^{n}$.

The following theorem gives us our final goal, provided $\operatorname{div} C \in L_{l o c}^{1}\left(\Omega, \mathbb{R}^{n}\right)$ and $\nabla^{s} \mathrm{C} \in L_{l o c}^{1}\left(\Omega, \operatorname{Sym}_{3}\right)$, that is, the corresponding distributions are represented by locally integrable functions.

Theorem 7. Let $P$ be a contact power of order 2 such that (c) of Definition 6 holds with $\mu_{1} \ll \mathscr{L}^{n}$ and $\mu_{2} \ll \mathscr{L}^{n}$ and let $\eta=\mu_{0}$. Assume moreover that $\operatorname{div} \mathrm{C} \in L_{l o c}^{1}\left(\Omega, \mathbb{R}^{n}\right)$ and $\nabla^{s} \mathrm{C} \in L_{l o c}^{1}\left(\Omega, \operatorname{Sym}_{3}\right)$.

Then there exists $h \in \mathcal{L}_{\text {loc },+}^{1}(\Omega)$ such that

$$
\begin{aligned}
P(M, v)=\int_{\partial_{*} M} v & {\left[(B-2 \operatorname{div} \mathrm{C}) \cdot \mathbf{n}^{M}+\left(\nabla^{s} \mathrm{C}\right) \mathbf{n}^{M} \mathbf{n}^{M} \mathbf{n}^{M}\right] d \mathscr{H}^{n-1} } \\
& +\int_{\partial_{*} M} \frac{\partial v}{\partial n}\left(\mathrm{C}^{M} \cdot \mathbf{n}^{M}\right) d \mathscr{H}^{n-1}+\int_{\partial_{*} M} v \mathrm{C} \cdot \mathrm{U} d \lambda_{M}
\end{aligned}
$$

for every $M \in \mathcal{C}_{h \eta}$ and $v \in C^{\infty}(\Omega)$.

In the same spirit as above, we show that the condition $\nabla^{s} \mathrm{C} \in L_{l o c}^{1}\left(\Omega, \operatorname{Sym}_{3}\right)$ has a counterpart in terms of $P$.

Theorem 8. We have that $\nabla^{s} \mathrm{C} \in L_{l o c}^{1}\left(\Omega, \operatorname{Sym}_{3}\right)$ if and only if there exists $h \in \mathcal{L}_{l o c,+}^{1}(\Omega)$ such that

$$
\left|\int_{\alpha}^{\beta} P\left(M_{s}^{(j)}, v\right) d s\right| \leqslant \int_{M_{\alpha, \beta}^{(j)}}\left(|v|+\left|\frac{\partial v}{\partial e_{j}}\right|\right) h d \mathscr{L}^{n}
$$

for every $\mathcal{M}_{G} \subseteq \mathcal{A}, M \in \mathcal{M}_{G}, v \in C_{c}^{\infty}(M), j=1, \ldots, n$ and $a_{j}<\alpha<\beta<$ $b_{j}$. In this case, we have $\left|\nabla^{s} \mathrm{C}\right| \leqslant \frac{3}{2}(h+|B-2 \operatorname{div} C|)$ on $\mathscr{L}^{n}$-a.a. of $\Omega$.

Acknowledgement. The research of the first author was partially supported by the MIUR project "Variational and topological methods in the study of nonlinear phenomena" (COFIN 2001) and by Gruppo Nazionale per l'Analisi Matematica, la Probabilità e le loro Applicazioni (INdAM).

The research of the second and third author was partially supported by the MIUR project "Modelli matematici per la scienza dei materiali" (COFIN 2002) and by Gruppo Nazionale per la Fisica Matematica (INdAM). 


\section{References}

1. M. Degiovanni, A. Marzocchi \& A. Musesti, Cauchy fluxes associated with tensor fields having divergence measure, Arch. Ration. Mech. Anal. 147 (1999), $197-223$.

2. M. Degiovanni, A. Marzocchi \& A. Musesti, Edge force densities and second order powers, submitted.

3. F. Dell'Isola, P. Seppecher, Edge contact forces and quasi-balanced power, Meccanica 32 (1997), 33-52.

4. A. Di CARlo, A. TAtone, (Iper-)tensioni \& equi-potenza, $15^{\text {th }}$ AIMETA Congress of Theoretical and Applied Mechanics (2001).

5. S. Forte, M. Vianello, On surface stresses and edge forces, Rend. Mat. (VII) 8 (1988), 409-426.

6. P. Germain, La méthode des puissances virtuelles en mécanique des milieux continus. Première partie. Théorie du second gradient, J. Mécanique 12 (1973), $235-274$.

7. P. Germain, The method of virtual power in continuum mechanics. Part 2: Microstructure, SIAM J. Appl. Math. 25 (1973), 556-575

8. M. E. Gurtin, L. C. Martins, Cauchy's theorem in classical physics, Arch. Ration. Mech. Anal. 60 (1976), 306-324.

9. M. E. Gurtin, W. O. Williams, W. P. Ziemer, Geometric measure theory and the axioms of continuum thermodynamics, Arch. Ration. Mech. Anal. 92 (1986), 1-22.

10. A. Marzocchi, A. Musesti, Decomposition and integral representation of Cauchy interactions associated with measures, Cont. Mech. Thermodyn. 13 (2001), $149-169$

11. A. Marzocchi, A. Musesti, Balanced virtual powers in Continuum Mechanics, Meccanica 38 (2003), 369-389.

12. W. Noll, E. G. Virga, On edge interactions and surface tension, Arch. Ration. Mech. Anal. 111 (1990), 1-31.

13. M. ŠILhAVÝ, The existence of the flux vector and the divergence theorem for general Cauchy fluxes, Arch. Ration. Mech. Anal. 90 (1985), 195-211.

14. M. ŠILHAVÝ, Cauchy's stress theorem and tensor fields with divergences in $L^{p}$, Arch. Ration. Mech. Anal. 116 (1991), 223-255.

15. W. P. Ziemer, Cauchy flux and sets of finite perimeter, Arch. Ration. Mech. Anal. 84 (1983), 189-201. 